\title{
Portable Virtual Endoscope System with Force and Visual Display for Insertion Training
}

\author{
Koji Ikuta Koji Iritani Junya Fukuyama \\ Department of Micro System Engineering, School of Engineering, Nagoya University \\ Furocho, Chikusa-ku, Nagoya 464-8603, Japan \\ TEL: +81 52-789-5024 FAX: +81 52-789-5027 E-mail: ikuta@mech.nagoya-u.ac.jp
}

\begin{abstract}
Drastically advanced "Portable Virtual Endoscope System" (Portable VES) with haptic ability is developed. This Portable VES can present not only computer graphical image of internal view from virtual intestine but also reaction force through the endoscope. Unlike previous VES reported in MICCAI'98, the portable VES has simpler drive mechanism with higher performance. Moreover, a large deformable dynamical model of both intestine and endoscope are formulated and implemented in the Pentium based a micro computer. Basic performance for clinical training is verified successfully by clinical surgeon.
\end{abstract}

\section{Introduction}

In today's medical clinics, low- invasive and non- invasive techniques of diagnosis and surgery that lighten the burden on the patient have come to be used frequently. A typical example is endoscopic diagnosis and surgery using an endoscope. However, there is no effective method of training medical personnel in these techniques, and there are reports of cases in which bodily organs have been harmed during diagnosis or surgery. To deal with this problem, in the last few years the authors have proposed and developed a virtual endoscope system (Fig. 1), with force sensation, to train young doctors and for simulation before surgery ${ }^{[1]},{ }^{[2]}$.

In a previous report ${ }^{[1]}$, we reported on functional improvements in the function of the force sensation display mechanism (VES-III) and the formulation of a real time dynamical model of an endoscope and intestine.

In the present work, we first designed and test produced a new type of force sensation display mechanism (VES-IV) that solves the problems that we had at the time of the last report, and performed a force sensation experiment. Next, in order to make possible training in endoscope insertion techniques under more realistic conditions, we developed a more sophisticated dynamical model of the endoscope and intestine. Then we displayed endoscope images and fluoroscopic images.

Finally, by linking the dynamical model and the mechanism we constructed a new system suitable for training in endoscope insertion. Here we report on an insertion experiment. 


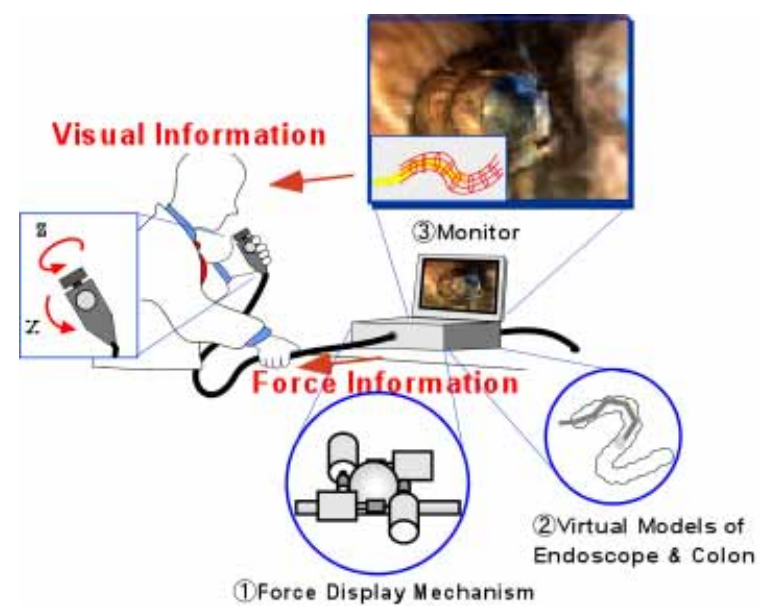

Fig. 1. Concept of virtual endoscope system with force sensation

\section{Design and Test Production of a Force Sensation Display Mechanism (VES-IV) Using the New Mechanism}

\subsection{Design and Test Production of the VES-IV}

A conceptual diagram of the new VES-IV is shown in Fig. 2. Rubber balls are placed at the center of a group of 4 rollers, and motor output is transmitted only through the rubber ball to drive both linear motion ball to drive both linear motion and rotation of the endoscope (this is called "Decoupled ball drive"). The test - produced VES-IV is shown in Fig. 3.

First, we summarize the principal features of the VES-IV.

1. Small and light weight

2. Improves responsiveness

3. Increased endoscope driving force and speed

4. Improved ability to hold the endoscope

5. Easy control

Now let us explain these features in detail.

1) As can be seen from Fig. 2 and Fig. 3, in the VES-IV the motor output is transmitted to the endoscope only through a hard rubber ball which has large surface friction; no gears are used. Therefore, compared to the VES-III, the transmission mechanism is very simple and efficient. At the same time great reductions in size and weight have been achieved.

2) In the VES-III, the motor output transmission mechanism was complicated and had many gears, so that gear backlash adversely affected the responsiveness. In contrast, in the VES-IV, as stated above, gears are not used; the simple transmission without gears, approaching direct drive, has eliminated backlash, improving responsiveness. 
3) Referring to Fig. 4(a), input T from the motor applies a downward force (1) and a moment (2) to the center of gravity of the rubber ball.Force (1) produces an increase in the vertical resistance force between the endoscope and the ball. At this time, the reaction to the frictional force between the endoscope and the ball produces the rightward force (3) on the center of gravity of the ball. This in turn increases the vertical resistance between the ball and the roller. Since the rubber ball will not slip against either the endoscope or the roller, the motor output is transmitted efficiently to the endoscope. Since the rubber ball is surrounded by 4 rollers, a similar effect occurs in all 4 directions during driving, producing an efficient driving force on the endoscope. The result is that the drive speed is 2.4 times that of the VES-III.

4) The VES-III had the problem that even within the force range expected to be encountered during the endoscope insertion operation, the endoscope slipped against the rollers, so that accurate force and position sensing could not be achieved. In the VES-IV, as shown in Fig. 4(b), the input force F produced by the endoscope insertion operation produces the rightward force (4) and the moment (5) on the center of gravity of the ball. At this time, contrary to the case discussed with regard to feature 3 ) above, first force(4) produces an increase in the vertical resistance between the rollers and the ball. At this time the reaction to the frictional force produces the downward force (6) on the center of gravity of the ball, increasing the vertical resistance between the endoscope and the ball, so that slippage does not occur as in 3). This effect resolves the problem that we had with VES-III producing a strong holding force without endoscope slippage even in case of a large input that exceeds the force anticipated in the endoscope insertion operation.

5) VES-IV uses 4 motors, 2 each to drive linear motion and rotation. However, since the output voltages applied to the 2 motors in each pair during driving are the same, control is as easy as it was for VES-III which had only 2 motors.

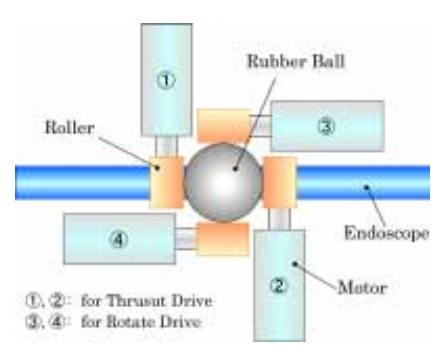

Fig. 2. Decoupled ball drive mechanism

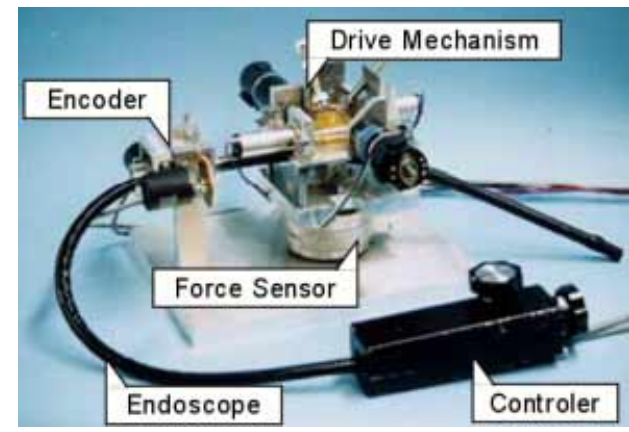

Fig. 3. Newly developed ompact force display mechanism using ball drive mechanism (VES-IV) 

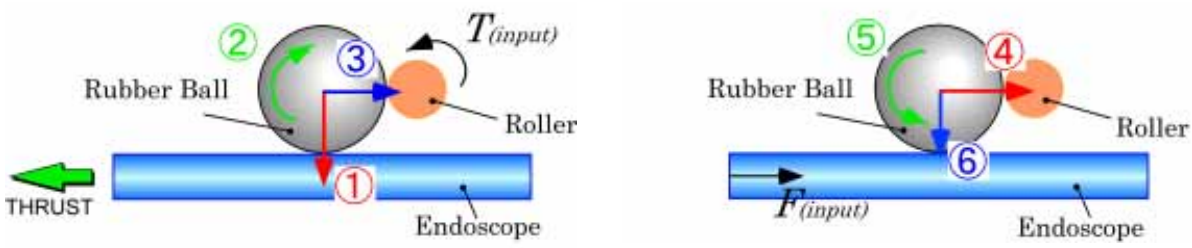

Fig. 4. Principle of a strong holding force by ball drive mechanism

\subsection{The VES-IV Force Sensation Display Experiment}

This system is required to provide feedback to the doctor that will provide a sensation similar to that encountered in an actual endoscope insertion operation. To determine whether it in fact ddes so, we did an experiment in which wedused the VES-IV system to express the sensation of resistance encountered during endoscope insertion in the virtual free condition and in the virtual "spring condition" representing the resistance encountered when the endoscope contacts, for example, the intestinal wall.

In the virtual free condition, control was applied so that the reaction force on the endoscope was always 0 . In the virtual free condition, the force read from a 6-axis force sensor was used to compute the theoretical position using a linear spring model; the motor output was determined from the difference between that theoretical position and the present position sensed by an encoder. The experiment results are shown in Fig. 5 and Fig. 6.

In the free condition in the forward motion direction (Fig. 5(a)), the error was held to the order of 10 [gf], a very good result. In VES-III, because of the mechanical problem discussed above it was difficult to obtain adequate drive speed, so that the reaction force oscillated to some extent. In VES-IV, adequate drive speed was obtained, leading to this good result.

In the rotation direction (Fig. 5(b)), waveform oscillations were compressed compared to the VES-III but were more obvious than in the forward motion direction. The cause of this was apparently that, in the VES-IV, for reasons of layout the gear motor free of backlash that was used to drive the forward motion could not be used to drive the rotational motion, so that there was an effect of backlash in the motor gear section. In the virtual spring condition, the VES-III had the problem that the endoscope slipped against the rollers, resulting in large reaction forces in both the forward motion and rotation directions, causing the motion to deviate from an ideal straight line. In the VES-IV good results along an ideal straight line were obtained for all spring constants in both the forward motion and rotation directions (Fig. 6(a), (b)).

\section{A Large Deformation Real Time Dynamical Model of an Endoscope and Intestine}

\subsection{Assumptions}

Since this system is for the purpose of training in endoscope insertion, as the doctor inserts the endoscope, the motions of the endoscope and the intestine must be analyzed, and force sensation information fed back to the doctor in real time. For this 
reason, analysis using the finite element method, which provides highly accurate solutions but requires a great deal of computation time, is unsuitable. Instead, the motions of the endoscope and the intestine were modeled in real time under the following assumptions.

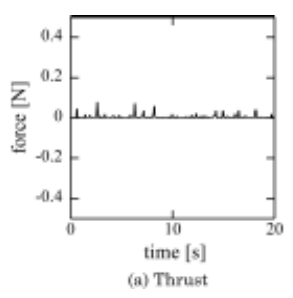

Fig. 5. Force display ability of VES-IV(free mode)

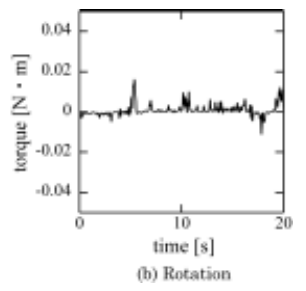

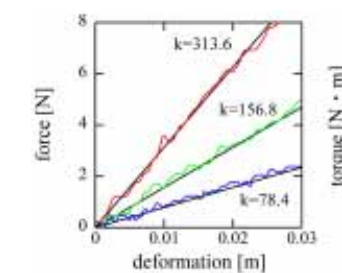

(a) Thrust

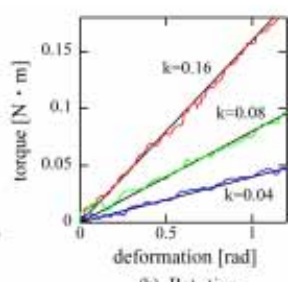

(b) Rotation

Fig. 6. Force display ability of VES-IV(elastic mode)

1. The endoscope is assumed to consist of a number of viscoelastic links, each compriding a rigid arm combined with viscoelastic components.

2. The intestine is assumed to be a viscoelastic circular cylinder of non-uniform inner diameter.

3. The reaction force is computed considering the contact force between the endoscope and the inside of the intestine.

First, we derived general equations of motion such as those that are used for manipulators. Then we neglected effects that are unimportant in considering the endoscope and intestine motions to simplify the equations.

\subsection{Modeling the Endoscope}

As stated, the endoscope is assumed to consist of n links, with (n-1) joints capable of viscoelastic motion. Each of the joints from the 1 st joint to the (n-2)nd joint is given 2 rotational degrees of freedom; the nearest, or (n-1)st, joint is assumed to have a thrust mechanism and a rotation mechanism with the same degrees of freedom as the mechanism section. Each joint is considered to be visco-elastic. Regarding the lengths of the links, in general the segments near the distal end of the endoscope are moved frequently and for long distances, and their direction of motion determines the path of advance of the whole endoscope. For this reason, the segments were made shorter toward the distal end, to improve accuracy, and longer as the root of the endoscope was approached (Fig. 7).

The motion of the endoscope modeled as described above was assumed to be represented by the Newton - Euler equations of motion, as follows.

$$
\begin{gathered}
\mathbf{M}(\mathbf{q}) \ddot{\mathbf{q}}+\mathbf{V}(\mathbf{q}, \dot{\mathbf{q}})=\sum_{i}^{n} \mathbf{J}_{r_{i}}^{T}(\mathbf{q}) \mathbf{F}_{i}+\mathbf{T} \\
\mathbf{T}=-\left|K\left[\mathbf{q}^{-} \mid \mathbf{q}_{0}\right]^{-}\right| D \dot{\mathbf{q}}
\end{gathered}
$$




\subsection{Modeling an Intestine Capable of Large Deformations}

\subsubsection{A Previously Reported Intestine Model}

The large intestine which we are considering is assumed to consist of a fixed section and a section which is practically not fixed at all. Since insertion into the section which is not fixed is difficult, there is a special insertion technique. For this reason, doctors having little experience can easily overextend the intestine when working in the section that is not fixed. This causes pain to the patient, and in the worst case damages the intestine. To reduce pain to the patient and make it possible to smoothly perform observations of the deep recesses of the intestine, practice in this special insertion technique is very important.

To make possible effective training and practice in the actual endoscope insertion technique, modeling of the section of the large intestine that is not fixed is indispensable. Until now, models of the intestine have only considered deformation of the intestine in the radial direction, and failed to consider the large deformations of the entire intestinal tube, referred to above, that are so important in training. Therefore, we here propose a new model of the intestine that considers large deformations of the intestinal tube itself.

\subsubsection{A Model That Considers Large Deformations of the Intestinal Tube Center Line}

As in previous models, the center line that determines the shape of the whole intestine is represented by equation (3).

$$
S(\mathbf{r})=0
$$

Designating the points into which the curve given by equation (3) is segmented by $\mathbf{r}_{\mathrm{i}}$

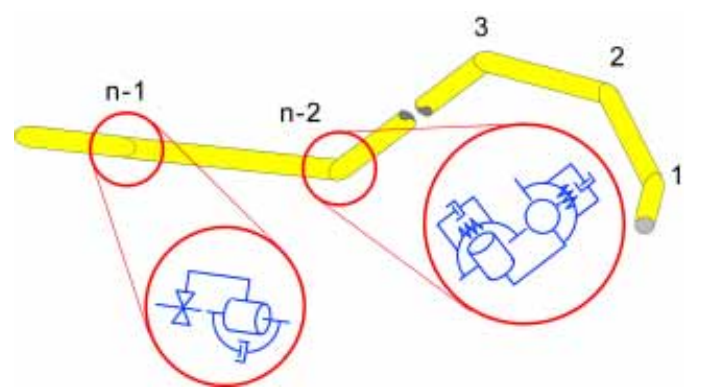

Fig. 7. Endoscope model with visco-elastic joints

(equation (4)), by using the circles of radii $R_{\mathrm{i}}$ (equation (5)) with those points as their centers, we form the 3 -dimensional tube of varying diameter. These circles lie on the planes $P_{\mathrm{i}}$ to which the lines tangent to the center line of the intestinal tube at the circle centers are normal.

$$
\begin{gathered}
\mathbf{r}_{i}=\left[\begin{array}{lll}
x_{c i} & y_{c i} & z_{c i}
\end{array}\right]^{T} \\
R=\phi(s)
\end{gathered}
$$

Distances from the center points are represented by the parameters. As shown in Fig. 8 , deformation of the intestine is modeled by displacing the segment points from their initial positions in response to loads applied by the endoscope with a visco-elastic model having an elastic coefficient $K_{\mathrm{c}}$ and a viscous coefficient $D_{\mathrm{c}}$. 
From the load received by a point contacted by the endoscope (equivalently, the magnitude of displacement of that point) the displacements of neighboring points are determined, leading to a model for the deformation of the entire intestinal tube in response to the load. However, to simplify the deformation of the intestinal tube, it is assumed that each of the points $\mathbf{r}_{\mathrm{i}}$ is displaced only on the corresponding plane $P_{\mathrm{i}}$. As in previous models, deformation of the intestinal tube in the radial direction is modeled with an elastic coefficient $K_{\mathrm{d}}$ and a viscous coefficient $D_{\mathrm{d}}$, and friction against the intestinal wall is modeled with a static friction coefficient $\mu_{\mathrm{s}}$ and a dynamic friction coefficient $\mu_{\mathrm{d}}$.

\subsection{Simplification of the Model}

In an actual endoscope insertion operation, both the endoscope motion and deformation of the intestine are very slow. Accordingly, in equation (1) the inertial terms as well as the centrifugal and Coriolis force terms are considered to be very small compared to the weight of the endoscope and the contact force, so that they can be neglected. As a result, equation (1) is simplified as follows.

$$
\mathbf{G}(\mathbf{q})=\stackrel{t}{i}^{n}, \mathbf{J}_{r_{i}}^{T}(\mathbf{q}) \mathbf{F}_{i^{-}}\left|K\left[\mathbf{q}^{-} \mid \mathbf{q}_{0}\right]^{-}\right| D \dot{\mathbf{q}}
$$

The motions of the endoscope and the intestine are analyzed by solving this equation of motion in short time steps. The viscous term for each movable joint has to be considered in view of the dynamical characteristics of an actual endoscope insertion tube. If these viscous terms are neglected, the time- dependent terms disappear, giving rise to unnatural behavior in which, if there is no contact force, at the next time step the joint angle returns to the balanced position.

\subsection{Judgment of Contact, and Computation of the Reaction Force}

To judge whether there is contact between the endoscope and the intestine, the distances between the endoscope model joints and the segment points of the intestine model are found, then the radii $R_{\mathrm{i}}$ of the intestine model at the segment points are compared geometrically.

In previous endoscope and intestine models, the intestinal tube itself did not deform, so to compute the reaction force it was sufficient to consider only contact between the endoscope and the intestine. However, this time the motion of the intestine itself is considered, so in computing the reaction force it is necessary to consider not only cases in which the endoscope contacts the intestine, but also cases in which the

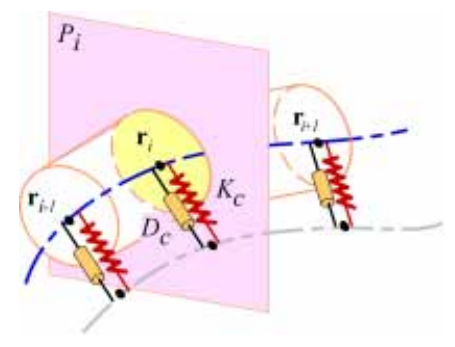

Fig. 8. Newly proposed colon model with visco-elastic suspentions

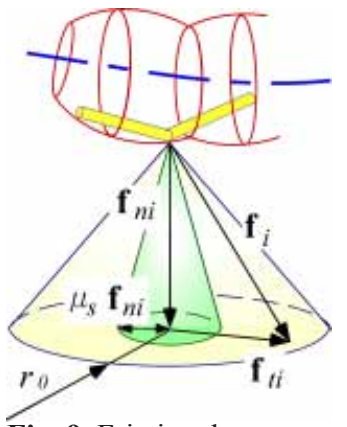

Fig. 9. Frictional cone 
intestine contacts the endoscope. As in previous models, friction is judged using a friction cone. To represent the effect of friction in movement along smooth contact surfaces, the friction cone shown in Fig. 9 is used. The inside cone represents the maximum static friction force; outside the outer cone (of radius $r_{0}$ ) is the dynamic friction region. $\mathbf{f}_{\mathrm{i}}$ is the contact force vector, with normal and tangential components $\mathbf{f}_{\mathrm{ni}}$ and $\mathbf{f}_{\mathrm{ti}}$, respectively.

First, the movable joints of the endoscope model and the segment points of the intestine model are given by equations (7) and (8), respectively.

$$
\begin{aligned}
& \mathbf{E}_{i}=\left[\begin{array}{lll}
X_{i} & Y_{i} & Z_{i}
\end{array}\right]^{T} \\
& \mathbf{r}_{i}=\left[\begin{array}{lll}
x_{c i} & y_{c i} & z_{c i}
\end{array}\right]^{T}
\end{aligned}
$$

1. Case in which the endoscope contacts the intestine

In the contact judgment, from the distance between the the endoscope joints and the intestine segment points, the direction vector $\mathbf{l}_{i}$, for the direction in which the endoscope presses against the intestine tube, is found. In the present model, the segment point displacement, that is, whether the intestine is deformed, is determined from the magnitude of $\mathbf{l}_{\mathrm{i}}$. The magnitude $\left|\mathbf{I}_{\mathrm{i}}\right|$ when deformation occurs is called $\mathrm{L}_{\mathrm{i}}$. If $\left|\mathbf{I}_{\mathrm{i}}\right|<=\mathrm{L}_{\mathrm{i}}$, it is judged that deformation does not occur. In this case, there are reaction forces on the endoscope from deformation of the intestine in the radial direction and from the attempt of the intestine segment point to return to its initial position. These correspond to $\mathbf{f}_{\text {ni }}$ in Fig. 9. $\mathbf{f}_{\text {ni }}$ is as given by the following equation. $K_{\mathrm{c}}, K_{\mathrm{d}}, D_{\mathrm{c}}$ and $D_{\mathrm{d}}$ are the viscous and elastic coefficients of the intestinal tube in the radial direction and the intestinal segment points, as discussed in section 3.3.2. $\mathbf{d}_{\mathrm{ri}}$ is the position vector of the segment point $\mathbf{r}_{\mathrm{i}}$ as seen from its initial position. The $1 \mathrm{st}$ term represents the force due to deformation of the intestinal tube in the radial direction; the 2 nd term the force exerted by an intestine segment point attempting to return to its initial position.

$$
\mathbf{f}_{n i}=\left\{\left|K_{d}\|\|_{i}\left\|\mid D_{d}\right\| \mathbf{i}_{i} \|\right\} \frac{\mathbf{l}_{i}}{\left\|\mathbf{I}_{i}\right\|}+\left\{\left|K_{c}\left\|\mathbf{d}_{\mathbf{r}_{i}}\right\|\right| D_{c}\left\|\dot{\mathbf{d}}_{\mathbf{r}_{i}}\right\|\right\} \frac{\mathbf{d}_{\mathbf{r}_{i}}}{\left\|\mathbf{d}_{\mathbf{r}_{i}}\right\|}\right.
$$

If $\left|\mathbf{l}_{\mathrm{i}}\right|>=\mathrm{L}_{\mathrm{i}}$, the intestine deforms, so fni is given by the following equation.

$$
\mathbf{f}_{n i}=\left\{\left\|K_{c}\right\| \mathbf{l}_{i}+\mathbf{d}_{\mathbf{r}_{i}}\left\|\mid D_{c}\right\| \mathbf{I}_{i} \|\right\} \frac{\mathbf{l}_{i}+\mathbf{d}_{\mathbf{r}_{i}}}{\left\|\mathbf{I}_{i}+\mathbf{d}_{\mathbf{r}_{i}}\right\|} j
$$

If the endoscope contacts the intestine, the contact takes place in the fi direction. First the angle $\mathrm{q}$ between $\mathbf{f}_{\mathrm{i}}$ and $\mathbf{f}_{\mathrm{ni}}$ is found, then the magnitude of $\mathbf{f}_{\mathrm{i}}$ becomes

$$
\left\|\mathbf{f}_{i}\right\|=\left\|\mathbf{f}_{n i}\right\| / \cos \theta
$$

then the tangential component $\mathbf{f}_{\mathrm{ti}}$ is found.

$$
\mathbf{f}_{t i}=\mathbf{f}_{i} \mid \mathbf{f}_{n i}
$$

2. Case in which the intestine contacts the endoscope

In this case, $\mathbf{f}_{\mathrm{i}}$ corresponds to the force of the intestine segment point trying to return to its initial position. At this time, $\mathbf{f}_{\mathrm{i}}$ is given by the following equation. The $\mathbf{d}_{\mathrm{ri}}$ are the respective position vectors of the segment points $\mathbf{r}_{\mathrm{i}}$ from their initial positions.

$$
\mathbf{f}_{i}=\left\{\left|K_{c}\left\|\mathbf{d}_{\mathbf{r}_{i}}\right\|\right| D_{c}\left\|\dot{\mathbf{d}}_{\mathbf{r}_{i}}\right\|\right\} \quad \frac{\mathbf{d}_{\mathbf{r}_{i}}}{\left\|\mathbf{d}_{\mathbf{r}_{i}}\right\|}
$$


In this case, if the direction of the tangential component $\mathbf{f}_{\mathrm{ti}}$ of $\mathbf{f}_{\mathrm{i}}$ is taken to be the direction of a link that has a movable joint $\mathbf{E}_{\mathrm{i}}$, then the angle $\theta$ between $\mathbf{f}_{\mathrm{i}}$ and $\mathbf{f}_{\mathrm{ti}}$ is found, the magnitude of $\mathbf{f}_{\mathrm{ti}}$ becomes:

$$
\mathbf{f}_{t i}=\left\|\mathbf{f}_{i}\right\| \cos \mathbb{E}
$$

and the normal component $\mathbf{f}_{\mathrm{ni}}$ is found as:

$$
\mathbf{f}_{n i}=\mathbf{f}_{i} \mid \mathbf{f}_{t i}
$$

Then, from the normal component $\mathbf{f}_{\mathrm{ni}}$ and the tangential component $\mathbf{f}_{\mathrm{ti}}$ of $\mathbf{f}_{\mathrm{i}}$ that have been found, $\alpha$, which expresses the friction condition, is found as in equation (16).

$$
f= \begin{cases}0 & \left\|\mathbf{f}_{i i}\right\|<\hat{\mathbb{E}}_{s}\left\|\mathbf{f}_{n i}\right\| \\ \frac{\left\|\mathbf{f}_{t i}\right\|-\hat{\mathbb{E}}_{s}\left\|\mathbf{f}_{n i}\right\|}{r_{0}-\hat{\mathbb{E}}_{s}\left\|\mathbf{f}_{n i}\right\|} & \hat{\mathbb{E}}_{s}\left\|\mathbf{f}_{n i}\right\|<\left\|\mathbf{f}_{t i}\right\|<r_{0} \\ 1 & \left\|\mathbf{f}_{t i}\right\|>r_{0}\end{cases}
$$

Then, using $\alpha$, the reaction force $\mathbf{F}_{\mathrm{i}}$ on the endoscope, seen from the reference coordinate system, is computed as in the following equation. The 1 st term is the static friction force component; the 2 nd term is the dynamic friction force component.

$$
\mathbf{F}_{i}=\left(1 \mid \ell \begin{array}{l}
\ell \\
\mathbf{f}_{i}+\ell
\end{array} \quad\left(\hat{\mathbb{E}}_{d}\left\|\mathbf{f}_{n i}\right\| \frac{\mathbf{f}_{t i}}{\left\|\mathbf{f}_{t i}\right\|}+\mathbf{f}_{n i}\right)\right.
$$

After the $\mathbf{F}_{\mathrm{i}}$ that are found in this way have been converted into the respective coordinate systems fixed in each of the movable joints using the conversion matrix, they are substituted into the endoscope model equation of motion given above.

\subsection{Analysis by Simulation}

Next, we performed actual analysis, using the dynamical model of an endoscope and intestine formulated as equations. Our endoscope model had 5 movable joints. As discussed in section 3.2, the 1 st to 4 th joints had rotating mechanisms with 2 degrees of freedom, while the 5th joint had a direct drive mechanism and a rotation mechanism with 1 degree of freedom each. The intestine model is given as a cubic center line divided into 150 segments. As discussed in section 3.3.2, each segment point was the center of a circle in the plane having a normal vector tangent to the intestine center line at the center point. In general the circles have different radii. An example of an analysis result obtained from the simulation will now be given.

We performed an analysis for the case in which an endoscope is inserted with constant force into a hypothetical intestine tube intended to model the sigmoid colon, a part of the large intestine that is practically not held in place at all. The motions of the endoscope and the intestinal tube in this case are shown in Fig. 10(a).

The reaction force toward the operator is shown in Fig. 10(b). The numbers in Fig. 10(b) have the same meanings as the corresponding numbers in Fig. 10(a).

As can be seen from the intestinal movement shown in Fig. 10(a), when the endoscope enters the sigmoid colon, the endoscope stretches the intestine considerably in the upward direction. It can be further seen from Fig. 10(b) that while this is happening the reaction force gradually increases. As can be seen particularly 
from (3) in Fig. 10(a), the operator seems to be inserting the endoscope at a constant rate, but as explained in section 3.3.1 at the distal end the endoscope stretches the intestine considerably, causing pain to the patient, and fails to penetrate into the deeper recesses of the intestine.

Previous intestine models, which only considered deformation of the intestine in the radial direction, gave simulation results showing that the distal end of the endoscope, while receiving a reaction force, penetrated deeply into the intestine ${ }^{[1]}$. The simulation results discussed above, on the other hand, show that when the considerable deformation of the center line, in addition to deformation in the radial direction, is considered, more realistic simulation results are obtained.

\section{Improving Visual Information}

\subsection{Required Specifications}

Doctor can obtain two kinds of visual information during endoscopic inspection into the intestine. Fig. 11 (a) is the endoscope image and (b) is the X-ray fluoroscopic image. The doctor relies primarily on the endoscope image when inserting the endo scope. An experienced doctor can judge from the color, shape and texture of the intestine. From this information and the distance that the endoscope has been inserted,
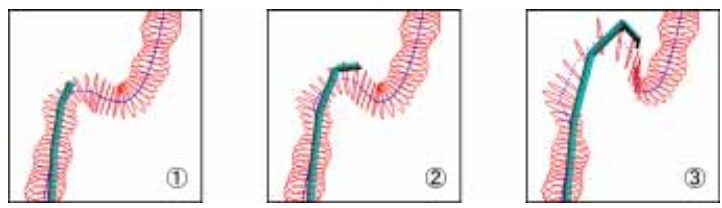

(a) Motion of endoscope model and colon model

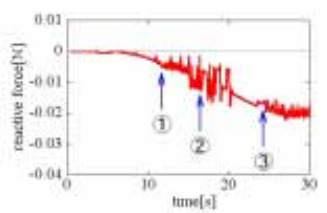

(b) Time-reactive force

Fig. 10. Simulation result

the experienced doctor forms an image of the shape of the endoscope insertion tube and is able to complete the technique in a short time without causing serious pain to the patient. It is indispensable that this endoscope image realistically shows the color, shape and texture of the intestine wall. An experienced doctor almost never uses the $\mathrm{X}$-ray fluoroscopic image.

However, a young doctor cannot form an image of the shape of the endoscope insertion tube just from the endoscope image. For this purpose he uses the X-ray fluoroscopic image. It is sufficient for the doctor to know the shapes of the endoscope insertion tube and the intestine, so it is not necessary to use the X-ray fluoroscopic 
image to Fig. 11(b). To provide better understanding of the shape, views are obtained from different angles while the endoscope is being inserted.

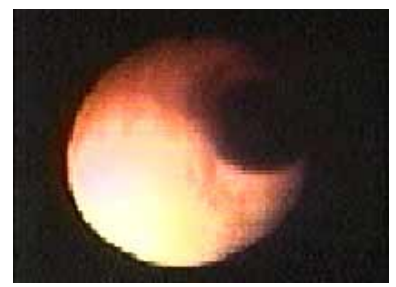

(a)Image of endoscopy

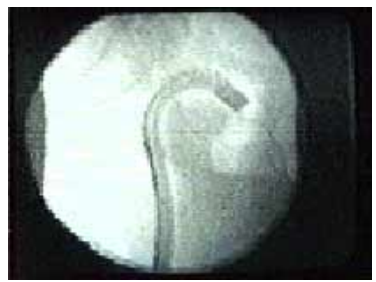

(b)Image of fluoroscopy

Fig. 11. Visual information during endoscopic operation

\subsection{Three Types of Endoscope Images}

As discussed in section 4.1, it is most important that an endoscope image realistically show the color, shape and texture of the intestine wall. In this system, realistic images are obtained by representing the intestinal tube model discussed in section 3.3 by a polygon model (Fig. 12(a)). The color and texture of each section of the intestine is mapped (Fig. 12(b)). In this way an experienced doct or can form an image of where the intestine the endoscope insertion tube is located. Fig. 12(c) shows an endoscope image using a wire frame. This is very effective in training beginners because it permits one to see parts of the intestine that cannot actually be seen.

Thus, it is possible to choose a method suitable for the doctor's skill level: a wire frame for training beginners, a polygon model for training intermediates, or texture mapping for training and surgery simulation for advanced physicians.

The method used can be changed while training is in progress, so that if the trainee becomes unable to form an image of the intestine it is possible to switch to a wire frame to increase the effectiveness of the training.

\subsection{X-Ray Fluoroscopic Images}

The two specifications required of X-ray fluoroscopic images were mentioned in section 4.1. In this system, the intestinal tube is represented by a sequence of rings (Fig. 13). This makes it easier to understand the shape of the endoscope inside the intestine and the contact situation.

Fig. 13 (a) and (b) show fluoroscopic images from different angles. To make the shape of the endoscope easier to understand, the angle of the fluoroscopic image can be varied continuously by mouse operation while training is in progress. In addition, a capability to enlarge the fluoroscopic image to show more detail is provided.

\subsection{The Total Image Display}

Fig. 14 shows an actual operation screen with both the endoscope image and the Xray fluoroscopic image incorporated into the system. In the control window at the upper left, the endoscope window can be selected, training repeated, damage to the 
intestine displayed and the intestine and endoscope to be used in the virtual space selected. These features were all created based on the requirements of the training system.

An input method that is easy for the user to understand intuitively has been adopted. This makes it possible for even people using the system for the first time to perform the various settings and operations.

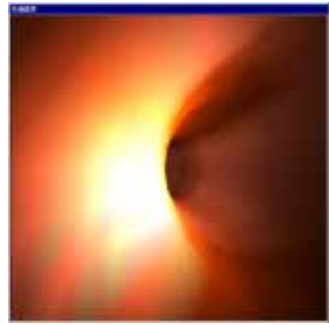

(a)Polygon model of intestine

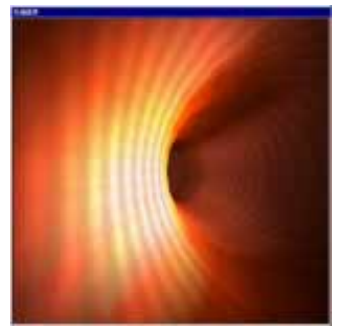

(b)Polygon model with texture mapping of real image

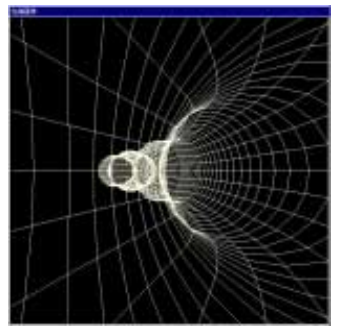

(c)Wire frame model of intestine

Fig. 12.Image of endoscopy for VES-IV

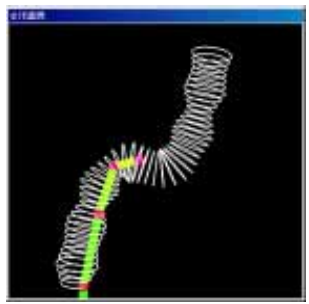

(a)

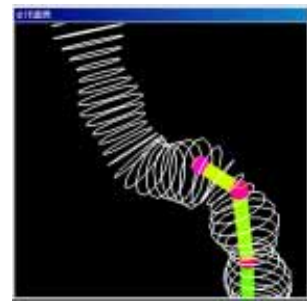

(b)

Fig. 13. CG image of fluroscopy for VES

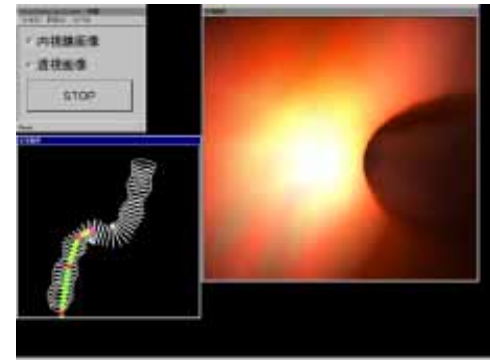

Fig. 14. Visual information for VES

Finally, in this system it is possible to choose whether to display or hide the X-ray fluoroscopic image. It has already been noted that an experienced doctor will generally not use the X-ray fluoroscopic image. However, the present reality that young inexperienced doctors are using X-rays on the patients. In other words, the patients are being exposed to X-rays at the time of observation. By hiding the X-ray fluoroscopic image while using this system, the doctor learns how to insert the endoscope into the patient without using the X-ray fluoroscopic image, making it possible to discontinue exposing patients to X-rays. Of course, the choice of whether to display or hide the image can be changed while training is in progress.

\section{Construction of an Endoscope Insertion Training System}

A system incorporating and linking the components that have been discussed thus far -- the VES-V, a dynamical model of the endoscope and intestine, and a fluoroscopic image display, into a system that can be used for endoscope insertion training, was constructed (Fig. 15). When an endoscope is inserted using the VES-V, the motions of 
the endoscope and the intestine are analyzed in real time and the reaction force and reaction torque are transmitted to the doctor's hand. In addition, a controller similar to the controller that will be used in an actual operation is used to adjust the angulation of the distal end of the endoscope to deform the intestine in a way that will decrease the reaction force, suppressing the pain felt by the patient as much as possible and making it possible to insert the endoscope deeply into the intestine.

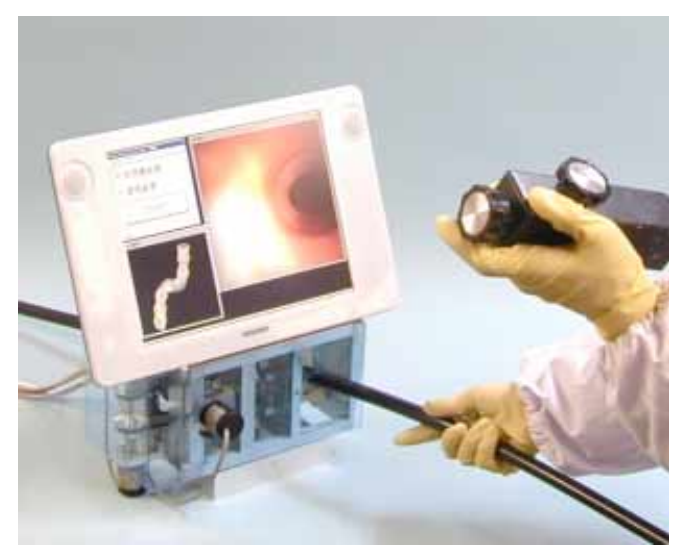

Fig. 15. Total virtual endoscope system with new force display mechanism and large deformable colon model (VES-V)

\section{Portable Virtual Endoscope System}

The latest version of the "portable virtual endoscope system" is shown in Fig.16. Our virtual endoscope system is constructed in three parts, a force display mechanism, virtual models of endoscope and colon, a monitor. In this portable virtual endoscpe system they are in a body of tower-type PC case. In recent years many surgical simulations are studied by various organization. But most of them isn't considered with portable ability. It is very important for training systems to be portable. Because many doctors need to practice high leveled insertion training. And the confidence of this system is progressed for using by many doctors. We made the very small force display mechanism which can be in PC case. Doctors can practice wherever there is a power supply.

As shown in Fig.17, the portable VES has a CD-ROM to read the personal data of patient's organ and the network ability also available to exchange data of the patient far from the trainee. It is easy to add new features according to demand.

\section{Summary}

Firstly, we clarify the problems that occur with mechanisms adopted in past research, and design and produce a new mechanism (the VES-IV) to solve those the problems that occur problems. The VES-IV was used to perform a force sensation display 


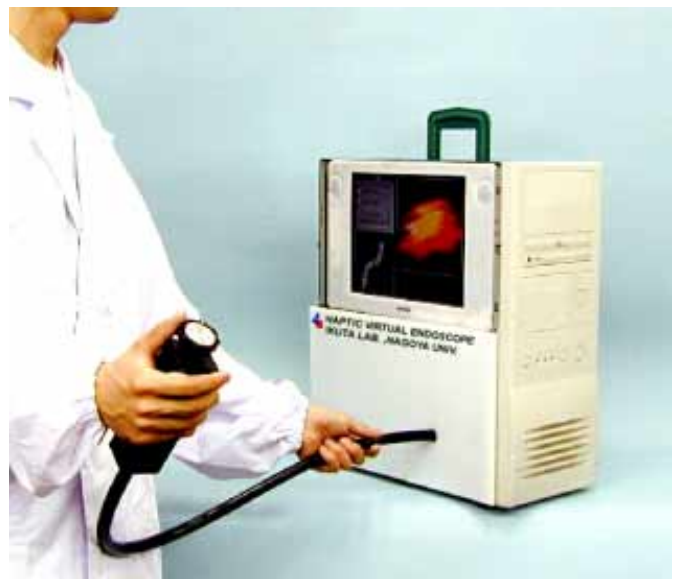

Fig. 16. Portable virtual endoscope system (VES-VI)

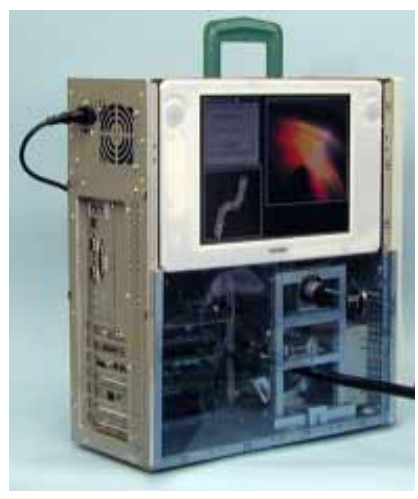

Fig. 17. Internal mechanism of Portable virtual endoscope system (VES-VI)

experiment, giving better results than have been obtained with past systems, confirming the effectiveness of this system. In the dynamical model, large deformation of the whole intestine that had been neglected in earlier models was considered. This model succeeded in simulating movements of the endoscope and the intestine close to those that occur in reality.

Both endoscope images and fluoroscopic images were provided, as in a real operation. To make the training system as complete as possible, several types of realistic endoscope images, and fluoroscopic images obtained from several angles, are provided.

Finally, the various elements were linked to construct a new system for realistic endoscope insertion training. And new portable training system was completed and performance was verified by the doctors.

Acknowledgment

Authors thank Mr. Shinkoh Senda and Mr. Masaki Takeichi of Nagoya University for his useful assistance for development.

\section{References}

1) K.Ikuta, M.Takeichi, T.Namiki: "Virtual Endscope System with Force Sensation",Medical Image Computting and Computer-Assisted Intervention (MICCAI 98), 1998

2)K.Ikuta, M.Takeichi, T.Namiki: "Virtual Endoscope System with Force Sensation",IEEE International Conference on Robotics and Automation ICRA99, pp.1715-1721, 1999 